\title{
Technical Considerations in using a Modified Outside-in Transforami- nal Endoscopic Discectomy Approach in Overcoming Lateral Recess Stenosis: A Case Study
}

\author{
Matthew Skoblar, DO ${ }^{1}$, Gabriele P. Jasper, MD², and Matthias H. Wiederholz, MD ${ }^{3}$
}

Microsurgery is a well-established and definitive surgical intervention performed for symptomatic radiculopathy secondary to lumbar disk herniation. While midline and paramedian Wiltse approaches have been the standard for lumbar nerve decompression, advances in neuroendoscopic instrumentation and visualization have broadened the ability of transforaminal endoscopic treatment to resect pathology in more challenging locations. Flexible instruments can be used to reach herniations in the epidural space as far centrally as the mid-vertebral body and enable physicians to reach cranially or caudally migrated sequestrations. Reviews of decompressive lumbar radiculopathy surgery have stated that there is equal efficacy when comparing the transforaminal endoscopic technique to standard microdiscectomy. However, questions have been posed by some authors in the literature regarding the utility of the endoscopic techniques with regard to patients with concomitant lateral recess stenosis and neurologic deficits.

The authors present a case of an endoscopically resected lumbar reherniated extrusion causing lateral recess stenosis and foot drop. The endoscopic approach is performed in an awakened patient with the use of local anesthetic. The optimal technique is described, including the use of the target, or bull's eye, view fluoroscopically to allow for enhanced accuracy when positioning the endoscopic working channel, as well as to allow for an increase in the intraforaminal space. The bull's eye approach is a more intuitive technique for placement of the endoscopic working channel for pain physicians as they are accustomed to navigating the foramen in this view. The use of an endoscopic approach with the initial surgery also allowed for the creation of minimal scar tissue, minimizing reoperative complication risks. The author's technique may offer a significant advantage in overcoming difficulties with lateral recess stenosis described in the literature and allow for more precise placement of the endoscope, particularly for pain physicians.

Key words: Endoscopic transforaminal discectomy, lateral recess stenosis, reherniation
Lumbar disk herniation is the most common cause of low back surgery with an estimated 200,000 surgeries performed annually in the United States (1). The pathophysiology involves a loss of disc nourishment via degeneration of vertebral endplates. This leads to fissuring and cracking within the lamellae of annulus

From : ${ }^{1}$ Department of Anesthesiology, Geisinger Medical Center, Danville, PA; ${ }^{2}$ Center for Pain Control, Brick, NJ; ${ }^{3}$ Performance Spine \& Sports Medicine, Newton, PA

Author for correspondence: Matthew Skoblar, DO

Address: Department of Anesthesiology, Geisinger Medical Center, 100 North Academy Avenue, Danville, PA 17822 E-mail: mmskoblar@geisinger.edu fibrils as well as desiccation of the nucleus pulposus, which herniates through the annulus often impinging on lumbar nerve roots. Lumbar discectomy surgery is often performed to obtain resolution of symptoms (1). One of the most common complications resulting from lumbar discectomy surgery is reherniation. Due to the variation in surgical approaches, types of annular defects, and measured durations of follow-up, the literature varies widely in its estimates of reherniation rates, with rates ranging from 5-24\% (1-3). Carragee et al (4) showed reoperation rates were higher if a > $6 \mathrm{~mm}$ annular defect was present and also showed an increased risk if there was an extruded fragment present with a large annular defect. Age, gender, 
the type of lumbar herniation, smoking, alcohol use, and the length of postoperative activity restriction are proposed risk factors within the literature, although more concrete research is needed (5).

There are many approaches a surgeon can take to remove a recurrent herniation including the open, microdiscectomy, and endoscopic approaches. Each approach has advantages and disadvantages although the literature states the reherniation rates of endoscopic discectomy are equivalent with the rates of open procedures $(1,2,6)$. The open and microdiscectomy approaches often involve incisions through muscles and tissues and removal of bone which may disrupt stabilizing structures and lead to significant development of scar tissue $(2,7)$. The endoscopic transforaminal approach involves less tissue destruction due to its posterior lateral trajectory and use of tissue dilation while allowing for a significantly larger increase in the intervertebral foraminal area (8). However, there have been questions in the literature regarding the efficacy of transforaminal endoscopic discectomy in patients with lateral recess stenosis, and certain authors have made strong recommendations that an open approach should be used in patients with accompanying neurologic deficits (9-11).

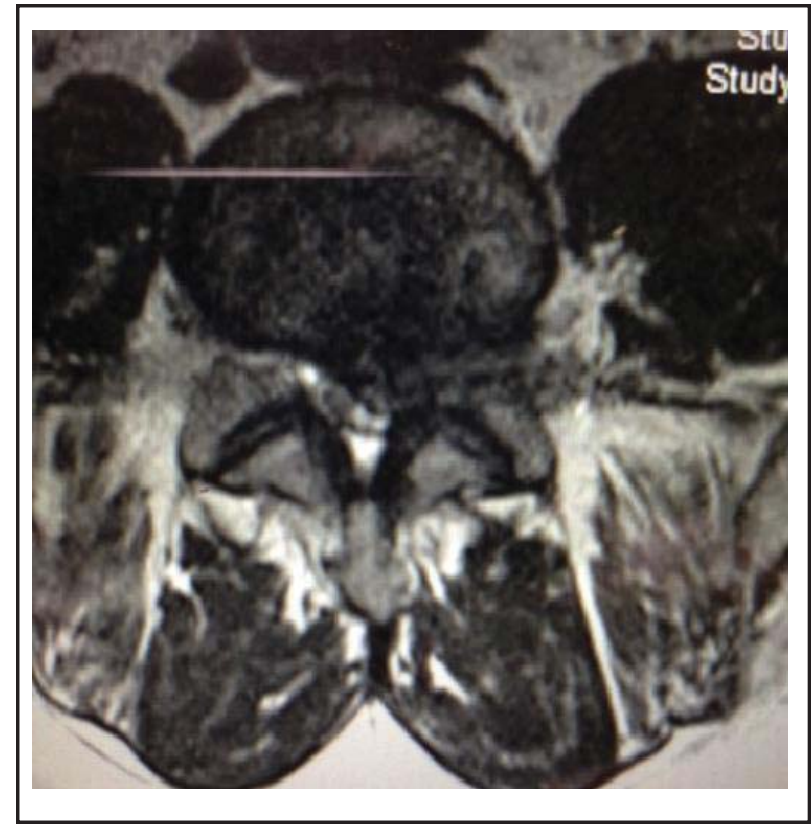

Fig. 1. Axial T2 weighted MRI slice at the L4-L5 disc showing reherniation and impingement on the exiting and traversing nerve roots.
The following is a case report detailing an optimal technique for the removal of a recurrent herniation in a patient with severe lateral recess stenosis and motor neurologic deficit that highlights the many advantages gained by using the author's modified transformational endoscopic approach.

\section{Case Report}

\section{History}

A 62-year-old man presented with a chief complaint of chronic low back pain, radiating to his left thigh and calf. The patient received treatments consisting of chiropractic care, physical therapy, and 2 transformational epidural steroid injections, with little relief. A review of magnetic resonance imaging (MRI) showed a large left L4-L5 paracentral disc extrusion with an annular fissure causing obliteration of the left lateral recess and compression of the traversing left L5 nerve root. The patient elected to undergo a transformational endoscopic discectomy. The initial procedure was performed without complication and immediate pain relief was noted. Three weeks postoperatively, the patient developed left foot drop after straining during a bowel movement. On a scale measuring strength and movement $(0=$ weakest,

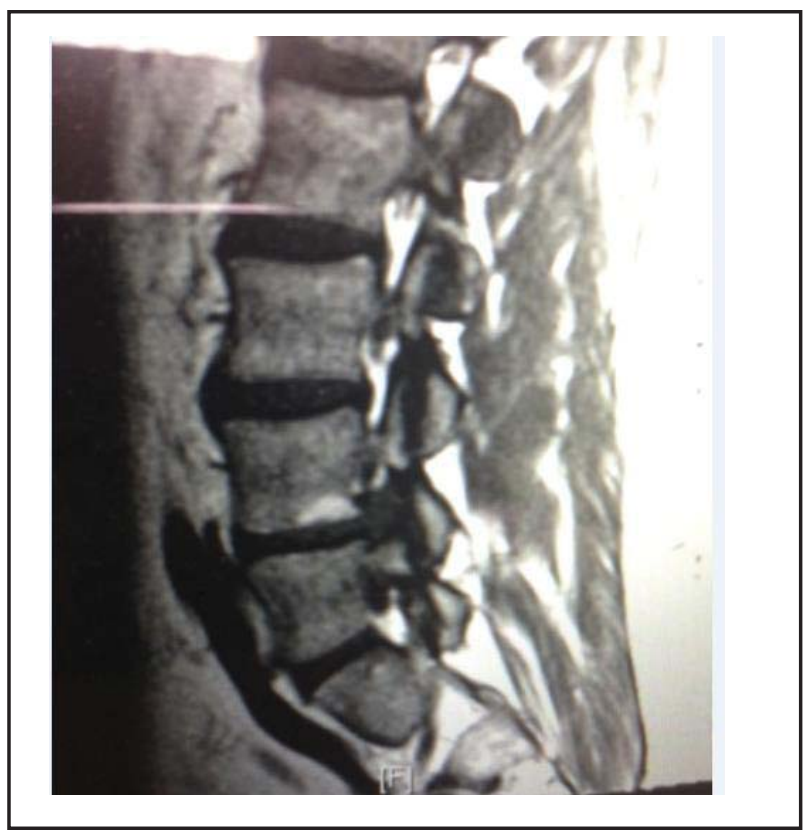

Fig. 2. Sagittal T2 weighted MRI showing L4-L5 reherniation within the neural foramen. 
$5=$ strongest), $3 / 5$ strength was noted in left ankle dorsiflexion and left great toe extension. His radicular pain had also recurred, but to a lesser severity. He denied bowel or bladder incontinence. Repeat MRI showed a large paracentral disk extrusion of the L4-L5 vertebral disk entirely compressing the lateral recess, with a new extruded fragment extending into the left neural foramen impinging on the $L 4$ nerve root and L5 traversing nerve within the L4-L5 neural foramen (Figs. 1 and 2).

\section{Operation}

The patient underwent a left L4-L5 transforaminal endoscopic discectomy. The patient was positioned in the right lateral decubitus position. A roll was placed under the flank to open up the disk space and lower the iliac crest, facilitating the entrance into the neural foramen. This exaggerated left lateral recumbent position cannot be emphasized enough, as it allows for better visualization of the neural foramen using a tunnel view. Anesthesia consisted of mild sedation using versed, fentanyl, and $1 \%$ lidocaine local anesthetic. The level of anesthetic was titrated so the patient was able to communicate with the surgeon throughout the procedure. The authors feel this is the safest way to prevent nerve injury as the patient can provide real-time feedback.

A joimax ${ }^{\circledR}$ TESSYS $^{\circledR}$ endoscopic system (joimax ${ }^{\circledR}$, Inc., Irvine, CA) was used for the procedure. The posterior-lateral trajectory was determined after reviewing MRI films, and entry was established at the L4-L5 foramen entering the skin about $12 \mathrm{~cm}$ lateral to the midline. Using anteroposterior (AP) and lateral fluoroscopy, as well as tunnel view, a $25 \mathrm{~cm} 18$ gauge needle was placed in the L4-L5 disk through
Kambin's triangle, between the exiting L4 nerve and the traversing L5 nerve. An AP fluoroscopic view was used to verify that the tip of the needle was not past the medial border of the pedicle. This ensured that the needle was not in the central spinal canal, thus avoiding the dural sac. A guide wire and dilator were used to negotiate the superior endplate or any bone spurs in the trajectory of the endoscope. This approach is essentially the Seldinger technique, using a flexible wire and small, curved dilator to negotiate the foramen so that sequential reamers can subsequently be utilized to enlarge the foramen. The beveled working cannula, $8 \mathrm{~mm}$ in outer diameter, was then placed over the sequential dilators. Rotating the beveled cannula and endoscope allows for a 360 degree direct visualization of the annulus and exiting and traversing nerve roots. The endoscope that was used had an optical angle of 30 degrees. The fluoroscopic endoscope placement is seen in Fig. 3. The beveled end of the working cannula was also used as a nerve root retractor.

The bipolar radiofrequency was used for further blunt dissection and hemostasis. The superior articular process (SAP) was identified, and the exiting nerve root was seen at its apex. The area was further decompressed by performing a partial facetectomy under direct visualization (Fig. 4). The exiting and traversing nerve roots were identified under direct visualization and were noted to be free of impingement and floating freely (Fig. 5).

\section{Postoperative Course}

At one week postoperatively, the patient no longer experienced any pain in his left lower extremity and had 3/5 motor strength in the extensor hallucis longus

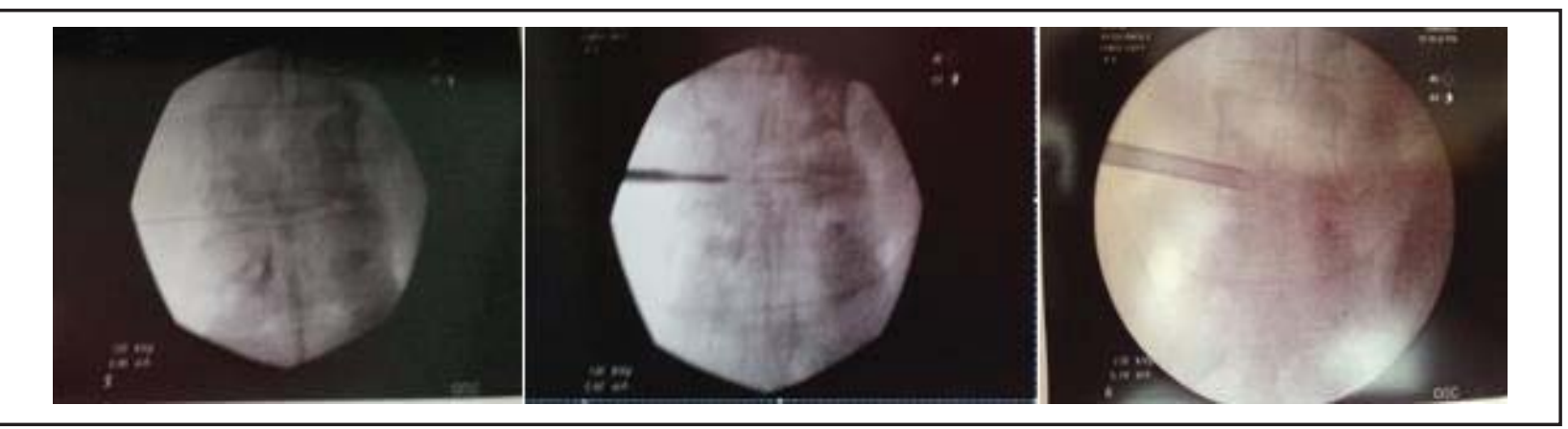

Fig. 3. An AP fluoroscopic view shows disk access and positive discography (left). Tubular dilators are used to gain access to the neural foramen and are confirmed not to be more medial than the medial border of the pedicle (middle). A view of the beveled working cannula (right). 


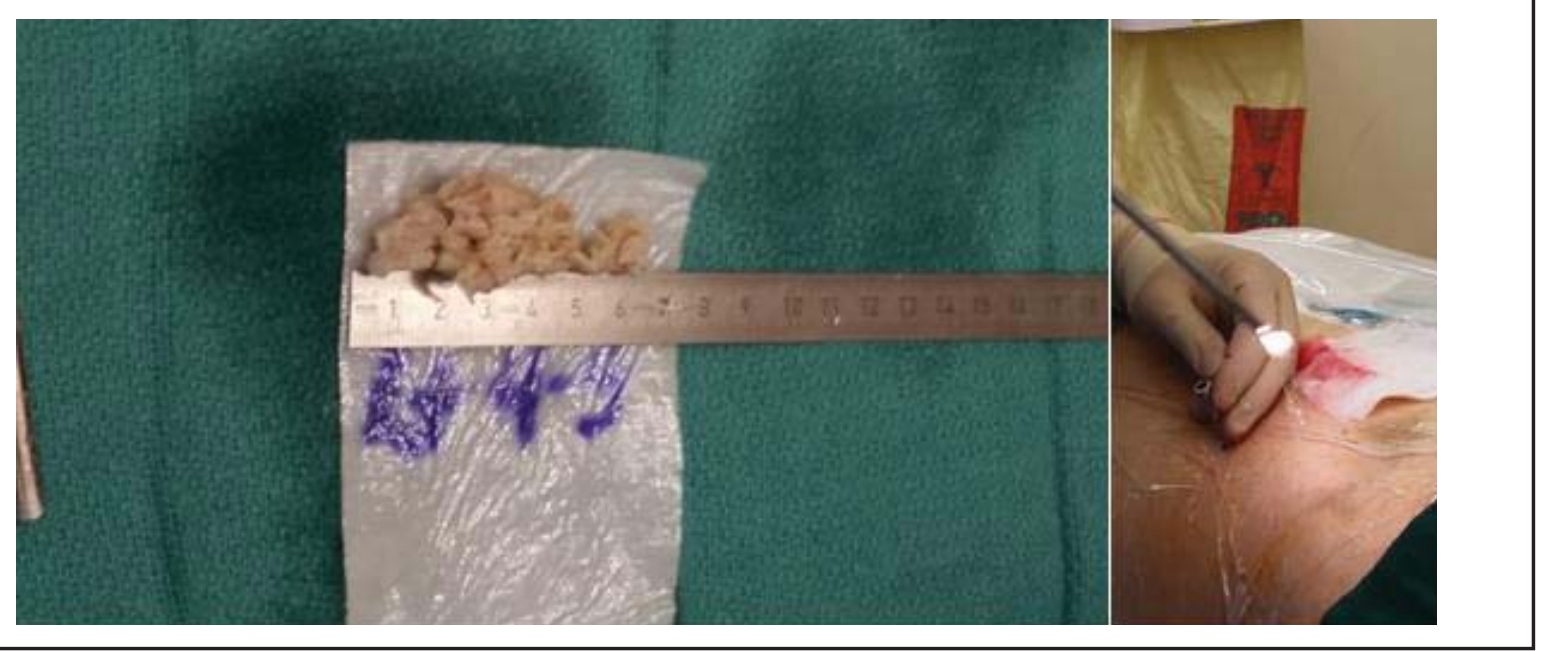

Fig. 4. The herniation was located under direct visualization, protruding from the annulus of the disc. Large and small portions of the herniation were removed with forceps graspers until the neural foramen was open enough for structures to be identified clearly.

(EHL) muscle. At 2 months, the patient remained pain-free with an improvement to $4 / 5$ strength in the left EHL muscle. At 7 months, the patient was symptom-free with resolution of foot drop.

\section{DISCUSSION}

Transforaminal endoscopic lumbar discectomy was introduced by Kambin in 1983 (12). Advances in endoscopic visualization and instrumentation have led to an increased popularity of the technique. Direct visualization of disk removal and foraminoplasty was made possible by the development of a uniportal approach (13). Additionally, endoscopes with a 30 degree optical angle have allowed for 360 degree visualization of the herniation, as well as the traversing and exiting nerve roots.

Concerning the transforaminal endoscopic discectomy technique, most of the literature describes an inside-out technique which involves the construction of an intradiscal working channel, which requires an annulotomy, prior to the removal of the herniation (9$11,13)$. In these reports, technical difficulty occurs in patients with high iliac crests, and worse outcomes are reported with the presence of lateral recess stenosis. However, the adaptation of a more lateral approach, as well as the use of specialized equipment, including sequential reamers and diamond-tip burr drills, have allowed for a targeted fragmentectomy to be performed in which the disk is not entered and an outside-in approach is used (8). The authors here have modified the technique further in a manner that is unique from other authors using the outside-in transforaminal endoscopic technique and feel that it may solve some of the difficulties mentioned above. The modification involves performing every procedure, regardless of the level, in a lateral decubitus position with a roll underneath the flank. This is advantageous for many reasons. It allows for:

- Further opening of the disk space and intervertebral foramen

- Rotation of the fluoroscope adequately enough to obtain a far lateral tunnel view, which allows more accurate entry into the lateral recess

- Lowering of the iliac crest and direct access to the lower lumbar and sacral neural foramen in patients with anatomically high lilac crests

- A theoretical decrease in the risk of complications arising from bleeding, due to a decrease in hydrostatic pressure, as there is less abdominal pressure and epidural engorgement in the lateral decubitus position as compared to a prone position

- A theoretical decrease in the risk of dural tear, as the dura is placed in a dependent position secondary to gravity

- Anesthetic advantages including better control of the airway and increased safety when deep sedation is necessary

- A more comfortable position for the patient. 

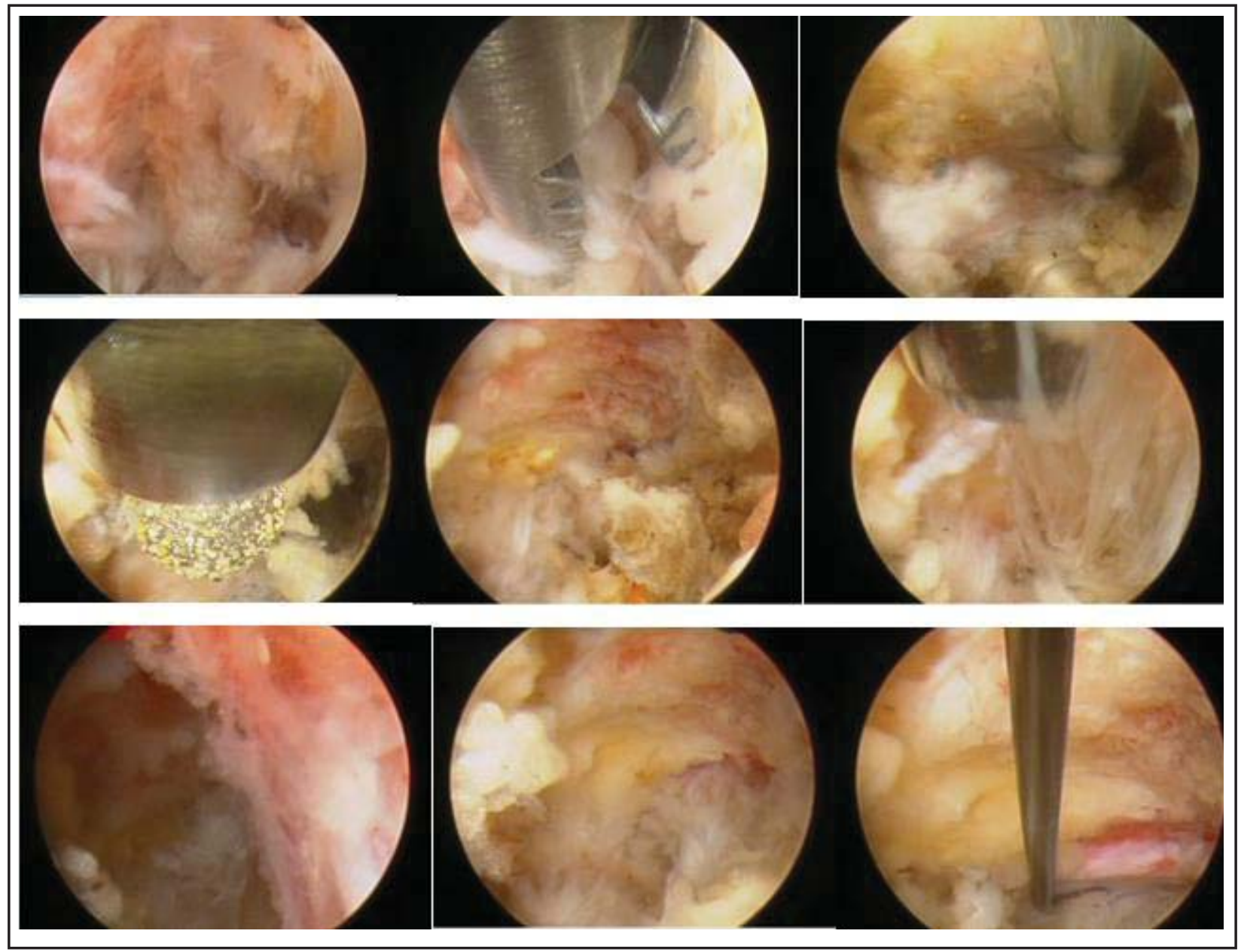

Fig. 5. Herniation is visualized with the TESSHSTM endoscope (top left) and removed with bendable graspers (top middle). The ligamentum flavum is visualized (top right), dissected, and then shaved down with a diamond-tipped drill (middle left). The lateral portion of the SAP is reamed and shaved down showing a smooth appearance (middle middle), which exposed more herniation (middle right). The exiting nerve root (bottom left) and the traversing nerve root (bottom middle) were identified and ensured to be floating freely (bottom right).

Poor results with lateral recess stenosis are quoted in recent literature (11) and large review articles (10), with some even listing it as exclusion criteria (8). However, many of these articles quote studies that are at least 10 years old as evidence, and 10 years ago the technique almost exclusively involved an intradiscal approach. For example, the 2015 literature review done by Kogias et al (10) quotes a study written by Ahn et al (9) in 2004 and describes difficulty with decompression in the lateral recess because "the horizontal part of the superior facet is more difficult to remove because it is thicker and harder than the more superior part." The use of a more lateral technique and the use of newer equipment including Kerrison rongeurs, diamond-tip burrs, flexible, curved graspers and drills, and endoscopic reamers equipped with a chisel allows for this obstacle to be more easily overcome. In this patient who had obliteration of the lateral recess with his first herniation and an extruded fragment in the lateral recess and foramen with associated foot drop in his second herniation, an effective outcome was obtained with the patient having complete pain relief after surgery. Foot drop was not present at the 7 month follow-up appointment. There was no difficulty in reaching the lateral recess in the original or revision surgery. 
When compared to standard discectomy approaches, the use of transforaminal endoscopic discectomy is shown to have similar reherniation rates $(1,6,14)$ with equally efficacious outcomes (10) following revision surgery. However, overall complication rates are reported to be $6 \%$ with endoscopic techniques versus $21 \%$ with microdiscectomy in revision operations (10). Using the transformational approach is advantageous because it allows for posterior ligaments, muscles, and bony structures to be spared, decreasing the risk for postoperative instability, disc space narrowing, and facet arthrosis $(2,7,8)$. Furthermore, the literature shows a very low rate of instability, which ranges from $0.4-4 \%$ with endoscopic transforaminal discectomy (10). This is logical due to the ability to access the foramen with little to no bone removal. In contrast, in revision operations using an open posterior decompression, a far lateral approach and/or wide facetectomy will have to be performed in order to sufficiently decompress a far lateral herniation or lateral recess stenosis, which would necessitate fusion due to the creation of instability (9). Lessened instability and tissue destruction may contribute to the observed decreased postoperative opioid use, the decreased need for hospital stays, a faster return to work, and a higher rate of return to athletic activities $(7,15)$. Interestingly, no differences in long-term satisfaction or pain scores are reported $(1,11)$.

Little to no scar tissue is created with the transforaminal technique, as it involves dilation of muscle and sparing of ligament and bone (7). This is pivotal when examining complications in reherniation procedures, as dissecting through dorsal scar tissue has been described as technically difficult $(2,7,16)$ and associated with increased rates of bleeding, infection, and neural injury $(2,3,7)$. Moreover, our authors believe the use of local anesthesia is also a key contributor in reducing the risk of neural injury while navigating through scar tissue, because patients are consciously sedated and can feel when the surgeon begins to engage material adjacent to the nerve root. Of note, there is less epidural scarring with the transforaminal endoscopic approach, because epidural vascular disruption is postulated to lead to more neural edema and sub- sequent epidural scar formation $(8,16)$. Reasons for less epidural venous bleeding can be attributed to the less destructive surgical approach and the use of pressurized saline solution for irrigation. Avoidance of epidural scarring is particularly important as formations of dural adhesions can lead to dural tear in subsequent operations and $10 \%$ of patients with scar tissue will be symptomatic and have chronic pain $(6,8)$ without many alternatives, as revision surgery for lysis of scar tissue adhesions is inadvisable by current literature (17).

Regarding the rate of dural tear in transforaminal endoscopic discectomy, there are no incidences of dural tear reported with the transformational endoscopic approach. There is $6 \%$ incidence in microdiscectomy (10). As mentioned above, the avoidance of creating and dissecting through scar tissue can decrease the risk of dural tear. With endoscopic approaches using dilation, it is possible to navigate the foramen through the same path initially used with minimal need for reaming, which limits the need to cut through vascularized scar tissue. In addition, the collapse of the transmusclar working channel after the removal of the equipment may further help minimize cerebrospinal fluid leakage. It is logical that the use of the lateral decubitus position may also help avoid dural injury as the dura is placed in a dependent position, further away from the working channel.

\section{CONCLUSION}

Endoscopic surgery is a relatively new and rapidly evolving method of disc removal that literature shows is as effective as microdiscectomy and standard open discectomy. It provides numerous benefits in terms of preserving anatomy and decreasing complication rates. New techniques have enabled more precise access to the foramen, and new tools have allowed for difficult-to-reach pathology to become accessible and treatable endoscopically. More study is needed to determine if the author's approach to lateral recess pathology is statistically significant, however, the intuitive nature of the bull's eye view and the outside-in technique may be worth adapting for pain physicians, despite the moderately high learning curve. 
Overcoming Lateral Recess Stenosis using Outside-in Transforaminal Endoscopic Discectomy

\section{REFERENCES}

1. Lee JK, Amorosa L, Cho SK, Weidenbaum M, Kim Y. Reccurent lumbar disk herniation. J Am Acad Orthop Surg 2010; 18:327337.

2. Kim CH, Chung CK, Sohn S, Lee S, Park SB. The surgical outcome and the surgical strategy of percutaneous endoscopic discectomy for recurrent disk herniation. J Spinal Disord Tech 2014; 27:415-422.

3. Hoogland T, van den Brekel-Dijkstra K, Schubert M, Miklitz B. Endoscopic transforaminal discectomy for recurrent lumbar disc herniation. Spine (Phila Pa 1976) 2008; 33:973-978.

4. Carragee EJ, Han MY, Suen PW, Kim D. Clinical outcomes after lumbar discectomy for sciatica: The effects of fragment type and annular competence. J Bone Joint Surg Am 2003; 85-A:102108.

5. Suk KS, Lee HM, Moon $\mathrm{SH}$, Kim NH. Recurrent lumbar disc herniation: Results of operative management. Spine (Phila Pa 1976) 2001; 26:672-676.

6. Ruetten S, Komp M, Merk H, Godolias G. Full-endoscopic interlaminar and transforaminal lumbar discectomy vs conventional microsurgical technique. Spine (Phila Pa 1976) 2008; 33:931939.

7. Ruetten S, Komp M, Godolias G. An extreme lateral access for the surgery of lumbar disc herniations inside the spinal canal using the full-endoscopic uniportal transforaminal approach-technique and prospective results of 463 patients. Spine (Phila $\mathrm{Pa}$ 1976) 2005; 30:2570-2578.

8. Choi G, Lee SH, Lokhande P, Kong BJ, Shim CS, Jung B, Kim JS. Percutaneous endoscopic approach for highly migrated intracanal disc herniations by foraminoplastic technique using rigid working channel endoscope. Spine (Phila Pa 1976) 2008; 33:E508-E515.

9. Ahn Y, Lee SH, Park WM, Lee HY, Shin SW, Kang HY. Percutaneous endoscopic lumbar discectomy for recurrent disc hernia- tion: Surgical technique, outcome, and prognostic factors of 43 consecutive cases. Spine (Phila Pa 1976) 2004; 29:E326-E332.

10. Kogias E, Franco Jimenez $\mathrm{P}$, Klingler JH, Hubbe $\mathrm{U}$. Minimally invasive redo discectomy for recurrent lumbar disc herniations. $J$ Clin Neurosci 2015; 22:1382-1386.

11. Kim MJ, Lee SH, Jung ES, Son BG, Choi ES, Shin JH, Sung JK, Chi YC. Targeted percutaneous transforaminal endoscopic diskectomy in 295 patients: Comparison with results of microscopic diskectomy. Surg Neurol 2007; 68:623-631.

12. Kambin P, Gellman H. Percutaneous lateral discectomy of the lumbar spine: A preliminary report. Clin Orthop Relat Res 1983; 174:127-132.

13. Yeung AT, Yeung CA. Advances in endoscopic disc and spine surgery: Foraminal approach. Surg Technol Int 2003; 11:255263.

14. Ruetten S, Komp M, Merk H, Godolias G. Recurrent lumbar disc herniation after conventional discectomy: A prospective, randomized study comparing full-endoscopic interlaminar and transforaminal versus microsurgical revision. J Spinal Disord Tech 2009; 22:122-129.

15. Birkenmaier C, Komp M, Leu HF, Wegener B, Ruetten S. The current state of endoscopic disc surgery: Review of controlled studies comparing full-endoscopic procedure for disc herniations to standard procedures. Pain Physician 2013; 16:335-344.

16. Cooper RG, Mitchell WS, Illingworth KJ, Forbes WS, Gillespie JE, Jayson MI. The role of epidural fibrosis and defective fibrinolysis in the persistence of postlaminectomy back pain. Spine (Phila Pa 1976) 1991; 16:1044-1048.

17. Ozgen S, Naderi S, Ozek MM, Pamir MN. Findings and outcome of revision lumbar disk surgery. J Spinal Disord 1999; $12: 287-292$ 